\title{
Development of IoT Health Monitor System using Security Patterns
}

\author{
E. R. Aruna, A. Rama Mohan Reddy, K. V. N. Sunitha
}

\begin{abstract}
Context: The most important non-functional requirement of the software application is the security. Developing Secure Software is a challenging Process. Software vulnerabilities and defects may disclose by developers, users, hackers due to Software-intensive systems get connected more and more in every day's lives. A better way to develop secure software is, enhance security processes in all the phases in SDLC. To enhance security in SDLC process required lots of mechanisms and systematic measures to assess the security during the development process. Objective: In this paper, we propose a method "Security aware-Software Development Life Cycle (Sa-SDLC) using Security Patterns". We also measure our security efforts in SDLC. This method fills the insecurity gaps from root level to top level in Granular style approach. Our method is suggestible for security critical applications such as Medical, Finance, Legacy and Communication (Messaging like email) Systems. Results: we successfully implemented our approach on remote health monitor since IoT devices are convenient in everyday life, these devices are using in home, environment, healthcare due to its feasible networking, storage and process features etc. In IoT health care applications, security of the sensitive data is paramount since humans are part of the IoT platform. IoTs heterogeneous network connectivity and expected growth, opens many new threats and attacks which impacts on life of a patient. Conclusion: Hence, our proposed methodology is implemented on Security Essential IoT based health care application and measures shows our method is improved software security.
\end{abstract}

Keywords: Sa-SDLC Methodology, Secure Patterns, IoT Application

\section{INTRODUCTION}

Internet becomes an integral part of everyone's life. Due to this, viruses, malicious actors, hackers, attackers increasing day by day, this badly affects vital information of the users. On the other hand Software Vulnerabilities are enabled due to implementation flaws or human weakness such as careless configuration, poorly chosen passwords. Hence Security must be injected into the application in all the software development phases such as requirements gathering and analysis, design, implementation, testing and deployment to achieve more secure software. Security must be an inbuilt part of iterative development process [1].

Revised Manuscript Received on February 05, 2020.

* Correspondence Author

E. R. Aruna*, Department of IT, Vardhaman College of Engineering, Hyderabad, India

A. Rama Mohan Reddy, Department of CSE, SVU Colege of Engineering, S.V University, India

K. V. N. Sunitha, Department of CSE, BVRIT College of Engineering, Hyderabad, India

(C) The Authors. Published by Blue Eyes Intelligence Engineering and Sciences Publication (BEIESP). This is an open access article under the CC BY-NC-ND license (http://creativecommons.org/licenses/by-nc-nd/4.0/)
Due to the lack of security measurement to quantify the performance, most of the software products are unsuccessful to produce protected environment for the end-users, still those products undergone through several formal methods and techniques for security. Security Process must be followed in consistent manner as per the procedures and rules throughout the SDLC. On the other hand, security measures such as metrics, frameworks, procedures, reviews etc. must use to quantity the efforts and progress [2].We can minimize the Software vulnerabilities by auditing and controlling every step in the software development Process [3].The Sa-SDLC method proposed several security mechanisms and measures to evaluate level of security in each process within the iteration and also after each development module.

Security Patterns mechanisms are used by information security engineers. There are vast number of patterns in the literature, to choose the appropriate pattern (Requirement phase) from the repository, model the pattern context (design phase) using security Modeling tools like secureUML, UMLsec etc. and implementation and validation of the pattern (development phase) and measure the efforts for security before and after applying these patterns are key functionalities in our work.

\section{Motivation towards Sa-SDLC using SPs methodology:}

Internet of Things (IoT) provides connectivity between the devices via wireless protocols, due to its heterogeneous potential the things get connected home, hospital, environment etc. The data through these devices must stored, managed and disposed whenever required. It is imperative to secure sensitive data since in remote health care applications humans are part of IoT Infrastructure. IoT devices low computation devices where as security algorithms are power hungry and computationally expensive. Hence we felt our Sa-SDLC methodology using security patterns is suitable for Security critical applications like IoT, cloud etc. The above problems are evaluated and measured using our proposed methodology.

\section{RELATED WORK}

Karen Goertzel et al discussed about existing security techniques, secure software in SDLC, secure SDLC principles, practices, security oriented requirements, Modeling for security capturing, Secure design principles and practices, secure design modes, Secure coding practices, Secure coding guidelines, security challenges, evaluating security[3]. 
Adel Mohammad et al evaluated three security top approaches McGraw's Touchpoints, Comprehensive Lightweight Application Security Process (CLASP) from Open Web Application Security Project (OWASP) organization, and Microsoft Security Development Lifecycle (SDL) with their process commonality, strengths, limitations and recognized cost, time and Lack of security knowledge are the reasons for security is not considered in software development. They suggested Adaptive Risk Framework and Automated Tool to enhance security activities within all SDLC phases and to consider security from the beginning of the project to the end [4].Anuradha Sharma et al compared existing 8 security techniques in the view of advantages and disadvantages for secure SDLC. All the techniques emphasized in design phase [5].

Measurement is a fundamental tool in Professional security management. Alfonso Bilbao et al proposed several parameters such as effectiveness, efficiency, performance, evolution of the threats, maturity to analyze security resources. They conclude security measure is the essential in risk management which focused on continuous improvement, they applied measures an example on "clothing store chain" [6]. Francis et al proposed attack graph analysis technique quantifies and compare the baseline state and attack surface after implementation of security framework. This technique also create quantitative measure allows to compare the efficiency of security framework across different applications [7].

Effective methods and tools must be used to evaluate software security and also Security performance must be measured in each process development iteration [8]. Minela Grabovica et al, analyzed about communication technologies such as RFID, Bluetooth, Wireless network and ZigBee and available measures for them In IoT. Finally discussed about the advantages of those technology measures [9]. Rizwana A.R. Shaikh et al proposed data security measurement tool is proposed to measure the data security and privacy of the cloud service provider. These security measurement enabled trust value parameter acts as checklist to be verified before selecting the cloud provider services [10].

Internet of Things (IoT) technological innovations impacts on economy in one hand, but the cyber risk of IoT impacts on the economy damage in other hand [11].

Stefano Tedeschi et al introduced an design approach for end point security in IoT connectivity to upgrade the legacy production machinery. This approach raised the future research includes mitigation mechanisms, systematic test generation and validation of solutions, automated risk assessment, impact of endpoint vulnerabilities, virtual isolation, systematic assessment and management of IoT security[12].

The major strengths of IoT: IoT is emerging in recent years with the capabilities of wireless protocols such as RFID, Wi-Fi, LTE etc. IoT promoting its potential with the performance of the protocols in terms of interoperability, scalability, reliability, quality of services, real-time, costeffectiveness etc. In the other side, security challenges such as privacy and confidentiality w.r.to these protocols need to be handled to get the full essence of the IoT [13]. Rob van Kranenburg et al stated the major weakness of Health care IoT(HIOT) is authorization, authentication, asset access control, real-time monitoring policies, access privileges and technical challenges [14]. Some of the open challenges like standardization, quality of health services, real-time monitoring, data storage space in implementing HIoT are acknowledged by S. M. Riazul Islam[15].

Security Pattern (SP) encapsulates reusable solutions for specific security problem. Hironori Washizaki et al [16] proposed taxonomy for security patterns, this taxonomy is used to select and use of the security patterns effectively. They prepared this SP taxonomy from research work on 200 papers about security patterns, attack patterns and misuse patterns. Continuous remodeling of systems for new deeds increases the complexity and more vulnerability, to avoid this problem, reliable and validated solutions are needed. Roberto Ortiz et al [17] suggested security patterns for this task, since these patterns provide documentation and solutions for recurrent security problems. Eduardo B. Fernandez et al[18] focused on core areas like cloud and IoT security. They have emphasized on secure architectures using the security patterns. They proposed future research scope on testing and empirically evaluating the security patterns to prove these pattern solutions are superior to other approaches.

Rest of the paper is organized as follows, section 3 presents the proposed Sa-SDLC methodology, section 4 implementation, section 5 presents results and discussion, and section 6 concludes the paper.

\section{THE PROPOSED METHODOLOGY}

We adapted Text Categorization Process [19], Restricted Misuse Case Modeling [20], validating security patterns using Test Template [21] approaches to extend our Methodology.

The proposed Sa-SDLC methodology, integrate security patterns mechanisms in each phase of SDLC to reduce impact of vulnerabilities. The block diagram Sa-SDLC is shown figure 1.

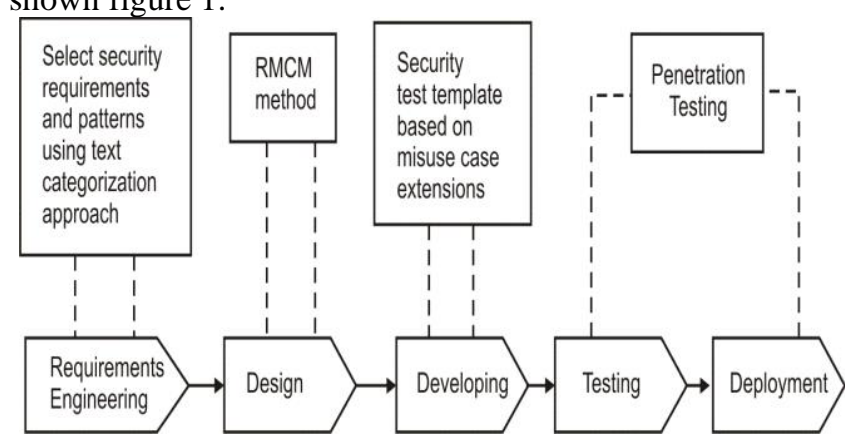

Figure 1: Block Diagram (Sa-SDLC using Security Patterns)

\section{The Steps in our Methodology:}

1. Select Functional and Security requirements of an application.

1.1. Identify Security Patterns for each Security Requirement from the pattern repository(To select the pattern,

we have used Text Categorization Process with Security Pattern Repository and Software Requirement Specification (SRS)).

Published By: 
2. Model the functional use cases along with security requirements and Pattern solutions using a Restricted Misuse Case Modeling (RMCM).

2.1. Elicit use cases with misuse case extensions diagram.

2.2. Derive the specifications for Using RMCM-V tool, check the consistency of diagram and specifications.

2.3. Elicit mitigation schemes (for specified threats in Misuse cases).

3. Develop the functional code and derive Test templates to support for mitigation of Misuse case.

3.1. Derive aspect test template based on Misuse case Extension

3.2. Use Test Driven Development (TDD) approach to create reusable Test case based on aspect test template.

4. Perform penetration Testing.

5. Deploy the Application with secure configuration.

\section{Goals of Sa-SDLC:}

- Projects Requirements stability

- Security ready product

- Reducing huge cost of post implementation for remediation.

As a justification, we applied "Sa-SDLC using Security Patterns" approach on Remote Health Monitor using IoT.

IoT Health Monitoring System: In figure 2, Health Monitor System Design, Patient bio-information like temperature, pulse rate, heart beat is captured through sensors. Sensor information is received by arduino. Through smart gateways patient data is transferred to cloud database from arduino. Remote care taker reviews the patient data through web application access.
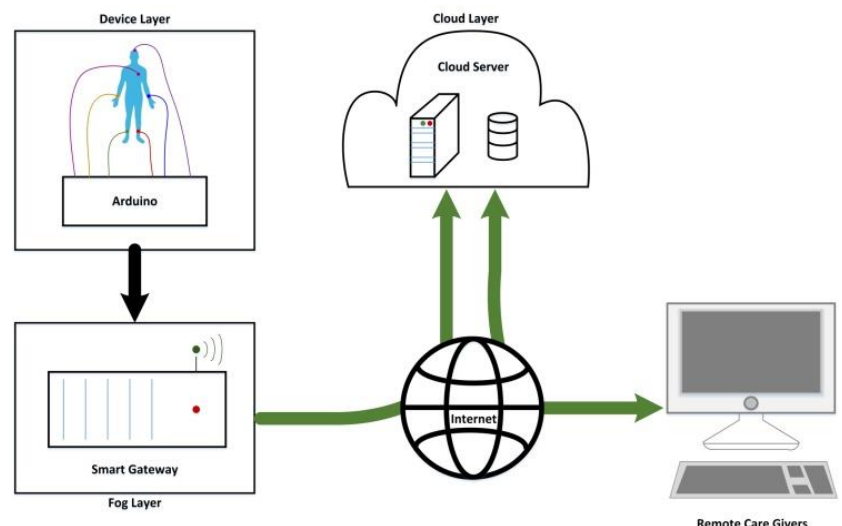

Figure 2: Iot Health Monitor System Design

\section{IMPLEMENTATION}

Step 1: Functional and Security Requirements of Remote Health Monitor System is

\section{Functional Requirements:}

1. This application enables, monitoring and tracking of Temperature, Heart rate of patient who is in remote location.

2. Sensors read Patient data, through arduino this data has to be transferred to Database using Wi-Fi.

3. The remote care takers (Doctor) reviews the patient data from time to time by accessing web application.

Security Requirements of Remote Health Monitor:
1. Arduino has to be authorized before storing data to the database.

2. Input data from trusted boundaries must be validated before process and grants access privileges.

3. Database has to be authenticated before store the data to the database.

4. Ensure the sensitive patient records remains confidential in transit, using encrypt forms. The sensitive data stored in database must be encrypted and also web application logon credentials needs to be encrypted and stored.

5. Access rights to be authorized before granting privileges to access the patient sensitive data by the remote care takers and all roles need to be defined and access rights for the records to be encapsulated.

6. Establish secure session, when user logged in and logged out. Web application Need to be invalidated the session when user is in inactive for certain period. Session Id must be unpredictable.

7. Provide maximum use of functionality to the users without presence of errors. But any security exceptions, while handling them not to disclose technical or sensitive details in simple error messages.

8. Need to be capture all logs and events, these audit records must be protected from being tampered.

9. Ensure the patient records do not alter/manipulate during usage of records by the malicious user.

Step 1.1 Identification of Security Patterns for Security Requirements: To identify the security patterns, Security Requirement Specification (SRS) and Security Pattern Repository (SPR) has to be prepared [19][22][23] as show in Table 1 and 2 respectively.

Part of structure of SRS Query:

\section{Table 1: SRS Query}

\begin{tabular}{|c|c|}
\hline SRS No & 1 \\
\hline SRS Name & Remote Health Monitor \\
\hline Domain & IoT \\
\hline Purpose/Scope & $\begin{array}{l}\text { Purpose: Design a Remote health Monitor } \\
\text { system. } \\
\text { Scope: Capturing patient data like heart beat } \\
\text { using sensors, Transit data } \\
\text { to cloud database storage and provide data to } \\
\text { remote viewing. }\end{array}$ \\
\hline \multirow{3}{*}{$\begin{array}{l}\text { Functional } \\
\text { Requirements }\end{array}$} & $\begin{array}{l}\text { This application enables, monitoring and tracking } \\
\text { of Temperature, Heart rate of patient who is in } \\
\text { remote location. }\end{array}$ \\
\hline & $\begin{array}{l}\text { Arduino and Sensors read Patient data, this data } \\
\text { has to be transferred to Database using Wi-Fi. }\end{array}$ \\
\hline & $\begin{array}{l}\text { Data collecting from Arduino, storing in the } \\
\text { database and presenting to remote care takers } \\
\text { done by web application }\end{array}$ \\
\hline \multirow{4}{*}{$\begin{array}{l}\text { Non-Functional } \\
\text { Requirements }\end{array}$} & $\begin{array}{l}\text { Arduino has to be authorized before storing data } \\
\text { to the database and Database has to be } \\
\text { authenticated before process and store the data. }\end{array}$ \\
\hline & $\begin{array}{l}\text { Access rights to be checked before granting } \\
\text { privileges to access the patient sensitive data by } \\
\text { the remote care takers. }\end{array}$ \\
\hline & $\begin{array}{l}\text { Establish secure session, when care taker logged } \\
\text { in and logged out. Web application need to be } \\
\text { invalidated the session when the care taker is } \\
\text { inactive for certain period. Session Id and other } \\
\text { session related details must be unpredictable. }\end{array}$ \\
\hline & $\begin{array}{l}\text { Provide maximum use of functionality to the } \\
\text { users without presence of errors. But any error } \\
\text { issues, error messages do not disclose technical } \\
\text { or sensitive details. }\end{array}$ \\
\hline
\end{tabular}




\section{Development of IoT Health Monitor System using Security Patterns}

\begin{tabular}{|l|l|}
\hline & $\begin{array}{l}\text { Need to be capture all logs and events, these } \\
\text { records must be protected from being tampered. }\end{array}$ \\
\cline { 2 - 3 } $\begin{array}{l}\text { All roles need to be defined and access rights for } \\
\text { the records to be encapsulated. }\end{array}$ \\
\cline { 2 - 3 } $\begin{array}{l}\text { Input data from trusted boundaries must be } \\
\text { validated before process and store. }\end{array}$ \\
\cline { 2 - 3 } $\begin{array}{l}\text { Ensure the sensitive patient records remains } \\
\text { confidential in transit, using encrypt forms. }\end{array}$ \\
\cline { 2 - 3 } $\begin{array}{l}\text { The sensitive data stored in database must be } \\
\text { encrypted and also web application logon } \\
\text { credentials needs to be encrypted and stored. }\end{array}$ \\
\hline
\end{tabular}

Part of Security Patterns Repository:

Table 2: Security Pattern Repository

\begin{tabular}{|l|l|l|}
\hline Pattern Id & $\# 1$ \\
\hline $\begin{array}{l}\text { Pattern } \\
\text { Name }\end{array}$ & Secure Directory \\
\hline Abstract & $\begin{array}{l}\text { Ensure attacker cannot alter the files during execution } \\
\text { of application }\end{array}$ \\
\hline Problem & $\begin{array}{l}\text { Attacker may alter/delete a file during the file data } \\
\text { relies on "unmodified", it causes "race condition". }\end{array}$ \\
\hline \multirow{5}{*}{ Solution } & $\begin{array}{l}\text { Canonical Path referred by Directory (contains files) is } \\
\text { secure (valid path), if and only if allow the file to } \\
\text { read/write. }\end{array}$ \\
\cline { 2 - 3 } Tradeoffs & Accountability & $\begin{array}{l}\text { Ensures the security for the } \\
\text { directory and files }\end{array}$ \\
\cline { 2 - 3 } & Availability & $\begin{array}{l}\text { Does not allow malicious } \\
\text { user to access file for } \\
\text { modification }\end{array}$ \\
\cline { 2 - 3 } & & $\begin{array}{l}\text { Files available only to the } \\
\text { users request through valid } \\
\text { paths }\end{array}$ \\
\cline { 2 - 3 } & Integrity & $\begin{array}{l}\text { Reliability of files in the } \\
\text { directory }\end{array}$ \\
\hline
\end{tabular}

Process of selecting the pattern from repository is shown in Pattern retrieval model in figure 3.

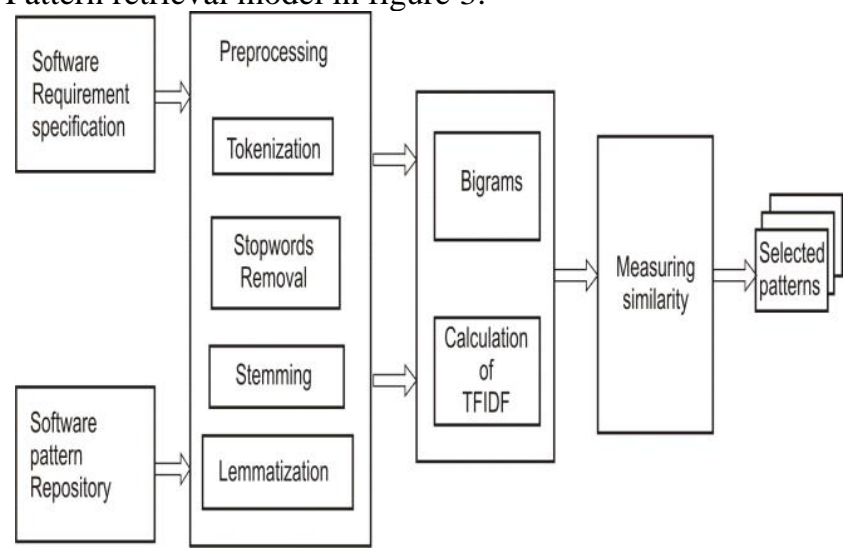

Figure 3: Pattern Selection Model

For SRS and SPR, preprocessing is done by performing Tokenization, stopwords removal, stemming, Lemmatization. and Bigrams are used to differentiate the security patterns. Singular Value decomposition (SVD) may apply for noise reduction and dimension reduction. Text categorization is done using Vector space model (VSM) Term-document category out of 3 VSM categories (other two are word-context and pair pattern document) for indexing. For SRS, indexing attributes are SRS No, SRS Name, purpose and Scope, Functional requirements, Nonfunctional requirements.

For SPR, the indexing attributes are Pattern Id, Pattern Name, Tradeoffs and single featured vector together Abstract, Problem and Solution. To know the weight of term, we used the filter based approach know as Term
Frequency Inverse Document Frequency (TFIDF) to remove the features.

The security design repositories have selected from the resources [24][25][26][27]. Based on the word similarity, the patterns resulted from Text categorization Approach shown in Table 3.

Table 3: Identified Patterns using Text categorization

\begin{tabular}{|c|l|}
\hline $\begin{array}{c}\text { Security Requirement } \\
\text { No in section IV. }\end{array}$ & \multicolumn{1}{c|}{ Security Pattern } \\
\hline 1 \& 3 & Secure Adapter Pattern \\
\hline 2 & $\begin{array}{l}\text { Input Validation Pattern and Password } \\
\text { Design and Use Pattern }\end{array}$ \\
\hline 4 & Secure Channels Pattern \\
\hline 5 & $\begin{array}{l}\text { Authorization Pattern , Role-based } \\
\text { Access Control Pattern }\end{array}$ \\
\hline 6 & Security Session Pattern \\
\hline 7 & Exception Manager Pattern \\
\hline 8 & Secure logger Pattern \\
\hline 9 & Secure Director Pattern \\
\hline
\end{tabular}

The prezaise context of pattern is analyzed with security requirements, this context provides a better communication between the requirements engineering to design. Throughout the next stages security requirements must be evaluated based on solution provided by security patterns.

Step 2. We model security requirements using the Restricted Misuse Case Modeling approach.

2.1 The part of usecase diagram with Misuse case extensions of IoT Health Monitor is shown in figure 4.

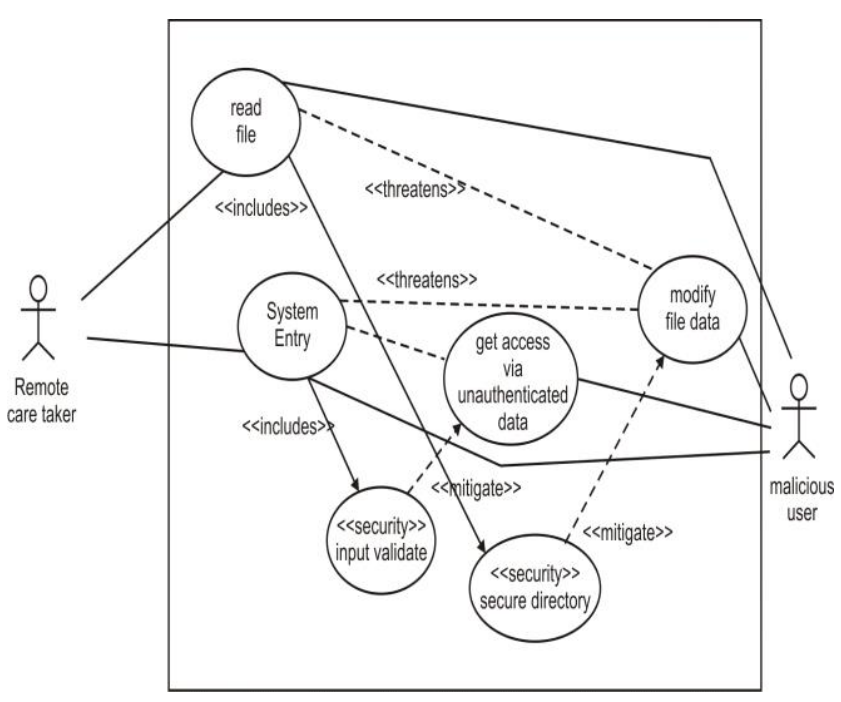

Figure 4: Misuse Case extension

2.2 The specifications for misuse case and security use case are shown in table 4 and table 5 as per the RMCM template. 
Table 4: Part of Misuse Case Specification

MISUSE CASE Get System entry via unauthenticated data

Precondition At least one web application user must registered

Primary Actor ATTACKER

Threats $\log$ in

Assets Patient sensitive DATA

Basic Threat Flow

1. The ATTACKER get system entry via unauthenticated(SQLi) or spoofing registered username and password

2. The username and password query evaluated by the system from Database

3. The system VALIDATES THAT, if query is successful, system sends welcome message to ATTACKER

Postcondition The attacker accessed Patient sensitive

DATA

Specific Alternative Threat flow

RFS 3

1. $\mathrm{DO}$

2. SENDS the error message to ATTACKER

3. The ATTACKER EXPLOITS error message

4. The ATTACKER PROVIDES SQLI VALUES

IN username and password UNTIL the query is successful

5. RESUME STEP 4.

Postcondition The ATTACKER access the Patient

DATA

Bounded Alternative Flow

RFS SATF1 1-4

1. IF the login attempts is reached to maximum THEN

2. Invalid login message send by the System TO the ATTACKER

3. ABORT

4. ENDIF.

Postcondition The ATTACKER cannot access Patient Sensitive DATA

Mitigation Scheme Secure Mechanism using Password

Design and Use Pattern

MISUSE CASE request for FILE to alter/delete

Precondition FILE exists the database

Primary Actor ATTACKER

Threats alter/delete the FILE

Assets FILE contains sensitive DATA

Basic Threat Flow

1. The ATTACKER requests the FILE, FROM the database.

2. The system grants access to FILE to ATTACKER.

Postcondition The ATTACKER obtained the FILE can ALTER/DELETE.

Specific Alternative Flow

RFS 2

1. IF the FILE path is INVALID

2. ABORT

3. ENDIF.

Postcondition The ATTACKER did not obtain the FILE DATA.

Mitigation Scheme Secure Mechanism using Secure Directory Pattern
Part of Security Use case Specification shown in Table 5.

Table 5: Security Use case Specification

SECURITY USE CASE FILE path Validation
Precondition at least one File existing in the system
Compliance System and application access control(ISO/IEC
27001:2013,clause A.9.4)
Mitigate access through unauthenticated DATA, Delete or
Modify File DATA
Basic Flow
1. The system receive the FILE request
2 . The system VALIDATES THAT the FILE
$\quad$ Path
Postcondition successfully validated Path using
canonicalization.
Specific Alternative Flow
RFS 2 The system displays an invalid FILE path.
ABORT.
Postcondition Error message is displayed.

Step 2.3: The mitigation schemes provided by Security Pattern solutions shown in table 6 as per the RMCM template

Table 6: Part of Mitigation Scheme from Pattern Solution

Scheme Name Secure Implementation of Remote Health monitor
based on IoT.
Brief Description This mitigate scheme, mitigates the
vulnerabilities for IoT based remote health monitor application
Actors Software Developer (Need not be a security specialist)
Mitigated Misuse Cases Expose sensitive data and access,
alter/modify the FILE DATA due to lack of authentication and
authorization
Compliance ISO/IEC $27001: 2013$ clauses A.6.1.5, A.9.4.A. A.10.1
Information security, access control and cryptographic controls
respectively.
Mitigation Tasks
1. VALIDATE input data from trusted boundaries with registered
data
2. ENCRYPT patient sensitive DATA and logon credentials of
input data.
3. VALIDATE roles before grant privileges for read/write
permissions.
4. Check path canonicalization before FILE granted
5.Apply selected security patterns solutions for the identified
specific problems

In the extended level, the consistency of the misuse case extension and its specifications may be verified by Restricted Misuse case Modeling -Verifier (RMCM-V) tool. Step 3: Develop the functional code and derive reusable Test template from mitigation scheme to support remediation of Misuse cases (vulnerability).

3.1 Create decision Tables and Pointcut Advice Model (Using Aspect Oriented Programming).

As per the Misuse case extension, prepared the decision table and pointcut advice pair to understand the internal processing of secure directory pattern is

The decision tables and Pointcut advice is shown in table 7 and figures 5, 6 .

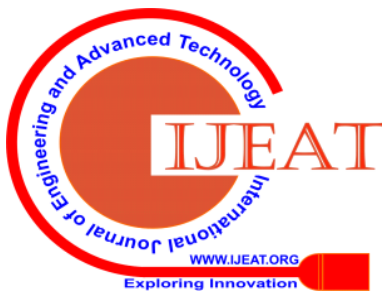


Development of IoT Health Monitor System using Security Patterns

Table 7: Decision Table of Security Directory Pattern

\begin{tabular}{|c|c|c|c|c|c|}
\hline & & 1 & 2 & 3 & 4 \\
\hline \multirow{2}{*}{$\begin{array}{c}\text { Conditi } \\
\text { ons }\end{array}$} & $\begin{array}{l}\text { Directory path for } \\
\text { requested File is } \\
\text { valid }\end{array}$ & Yes & Yes & NO & NO \\
\hline & $\begin{array}{l}\text { User permission } \\
\text { to access the File } \\
\text { is true }\end{array}$ & Yes & No & Yes & No \\
\hline \multirow{2}{*}{ Actions } & $\begin{array}{lr}\text { File can } & \text { Access } \\
\text { from } & \text { the } \\
\text { Directory } & \end{array}$ & & X & X & $X$ \\
\hline & $\begin{array}{l}\text { Deny to access } \\
\text { the File from the } \\
\text { Directory }\end{array}$ & X & & & \\
\hline
\end{tabular}

pointcut validateSecureDirectory 0

call(**.Secure_Directory.checkSecureDirectory (..);

call (**.Secure_Directory.validatePath(..) ;

Figure5: Pointcut for validating directory

after 0 returning(Boolean right):

Permit_accessfile( $\{$ setTemporary ("validateSecureDirectory",right);

Figure 6: advice to provide file access from the directory

3.2 Create reusable Test case based on Test Driven

Development (TDD) approach using 3.1.

Part of the test case template is shown in figure 7 derived from Security Directory Pattern. This test case is reusable. This will support the pattern validation during development phase. The developers may not have security knowledge can use this Template.

Step 4: The application, Penetration Test has done and verified the functional and security requirements. The security penetration is conducted dynamically. One Test case results is shown in figure 8.
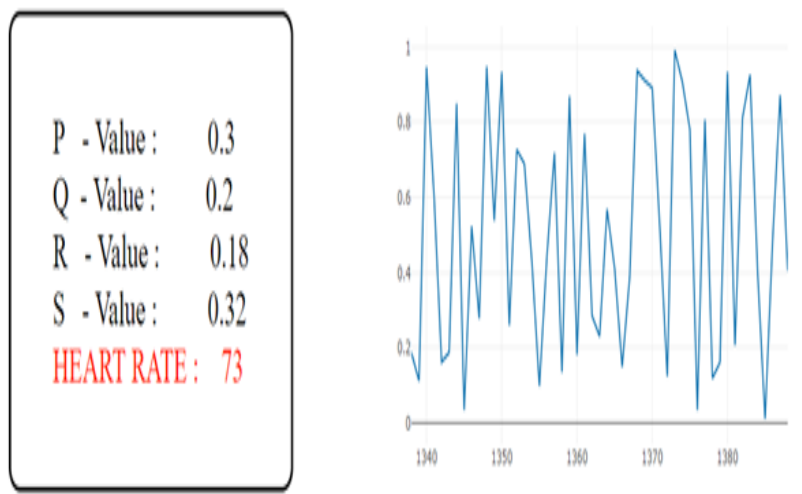

Figure 8: Heart Rate of Patient package secureDirectoryPattern;

import org.junit.Before;

import org.junit. Test;

import secureDirectoryPattern.SecureDirectorRepository.Permission;

import static org.junit.Assert.assertEquals;

import java.io.BufferedReader,

import java.io.FileReader;

import java.io.IOException

public class SecureDirectoryCheck

private static SecureDirectorRepository repository $=$ null;

private static String userId, userRequestfilePath;

private static Permission requestAccess;

$@$ Before

public void setUp0 throws Exception \{

repository = new SecureDirectorRepository0;

setUserData("uttej","D:\data\\ARUNA \log.txt",

Permission.READ);

@Test

public void validateUser 0 \{

assertEquals(repository usersMap.containsKey(userId), true);

assertEquals(repository.permissionsMap.containsKey(repository.user

sMap.get(userId)), true);

String user = repository.usersMap.get(userId);

if (repository.permissionsMap.get(user) $=$ requestAccess) \{

try \{

String data;

StringBuilder dataBuilder = new StringBuilder0;

@SuppressWarnings("resource")

BufferedReader $\mathrm{br}=$ new BufferedReader(new

FileReader(userRequestfilePath));

while $(($ data $=$ br.readLine $)) !=$ null $)\{$

dataBuilder.append(data); $\}$

System out.println(dataBuilder.toString 0 );

catch (IOException e) \{

e.printStackTrace);

\} finally \{\}$\}$

else \{throw new RuntimeException("Unauthorized!!!");\}\}

public void setUserData(String id, String filePath, Permission

permitAccess) \{

userId $=$ id; userRequestfilePath $=$ filePath;requestAccess =

permitAccess;

\})

Figure 7: Part of Reusable Test Case Template

The test case using Security Directory Pattern solution is shown in figure 9.

$\begin{array}{lll}\text { Finished after } 0.019 \text { seconds } & \\ \text { Runs: } 1 / 1 & \text { W Errors: } 0 \quad \text { a Failures: } 0\end{array}$

S. secureDirectoryPattern.SecureDirectory Test [Runner: JUnit 4] (0.001 s)

Step 5: The application is deployed with secure configuration, such as use of legacy equipment, standard devices and time to time OS updation.

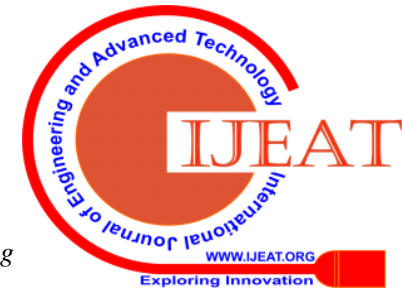


At this phase the risk is low profile, simple remedies like disable auto-run features without authorization, remove unnecessary accounts, authenticate is mandatory before access data, software uninstall if they are unnecessary etc.

\section{RESULTS AND DISCUSSION}

Based on the methodology, the developed code exhibits the following results shown in table 8 .

Table 8: PQRS complex values and Heart Beat of 5 Patients

\begin{tabular}{|c|c|c|c|c|c|}
\hline Patients & $\mathbf{P}$ & $\mathbf{Q}$ & $\mathbf{R}$ & $\mathbf{S}$ & Heart beat rate \\
\hline Patient 1 & 0.02 & 0.009 & 0.6 & 0.06 & 71 \\
\hline Patient 2 & 0.14 & 0.12 & 0.65 & 0.05 & 70 \\
\hline Patient 3 & 0.002 & 0.001 & 0.82 & 0.123 & 109 \\
\hline Patient 4 & 0.2 & 0.31 & 0.92 & 0.287 & 80 \\
\hline Patient 5 & 0.4 & 0.091 & 0.88 & 0.282 & 89 \\
\hline
\end{tabular}

Whenever sensors are attached to the patient body, automatically secure code runs to capture the data and transfer to database with secure communication. Doctor can see and review the patient condition from anywhere after authenticated through web application.

\section{CONCLUSION}

In literature, selection and application of security patterns for security requirements of IoT does not have empirical methodology. The limitation in [21] may overcome i.e Object Constraint Language (OCL) may not necessary to prepare Decision Table and pointcut advice Model (Aspect Programming) to understand the pattern internal processing. Instead of OCL, we can use Misuse case extension to prepare decision table and Pointcut Advice pair model.

Using Sa-SDLC, we successfully integrated security in each phase using security patterns. This Sa-SDLC is most suggestible for security critical Applications. We believe, security patterns are the powerful tool for Secure SDLC.

\section{REFERENCES}

1. Pieter Frijns,Robert Bierwolf,Tom Zijderhand," Reframing Security in Contemporary Software Development Life Cycle", IEEE International Conference on Technology Management, Operations and Decisions (ICTMOD), 2018.

2. Nunes FJB, Belchior AD, Albuquerque AB. "Security engineering approach to support software security. In SERVICES. 2010", July; 48-55.

3. Karen Mercedes Goertzel, Booz Allen Hamilton," Enhancing the development life cycle to produce secure software", Software Assurance Forum, 2008.

4. Adel Mohammad, Ja'far Alqatawna, Mohammad Abushariah," Secure Software Engineering: Evaluation of Emerging Trends", 8th International Conference on Information Technology (ICIT),IEEE,2017.

5. Anuradha Sharma, Praveen Kumar Misra," Aspects of Enhancing Security in Software Development Life Cycle",Advances in Computational Sciences and Technology, 2010.

6. Alfonso Bilbao, Enrique Bilbao," Measuring Security", 47th International Carnahan Conference on Security Technology (ICCST), IEEE, 2013.

7. Francis J. Manning, Frank J. Mitropoulos, Utilizing Attack Graphs to Measure the Efficacy of Security Frameworks Across Multiple Applications", 47th Hawaii International Conference on System Science,IEEE,2014.

8. Shehab A. R. Farhan and Mostafa G. M. Mostafa," A Methodology for Enhancing Software Security During Development
Processes",21st Saudi Computer Society National Computer Conference (NCC), 2018.

9. Minela Grabovica, Dražen Pezer," Provided security measures of enabling technologies in Internet of Things (IoT): A survey", Zooming Innovation in Consumer Electronics International Conference (ZINC), IEEE,2016.

10. Rizwana A.R. Shaikh, Masooda M. Modak, "Measuring Data Security for a Cloud Computing Service", International Conference on Computing, Communication, Control and Automation (ICCUBEA).2017.

11. Petar Radanliev, Dave De Roure, Stacy Cannady, Rafael Mantilla Montalvo, Razvan Nicolescu, Michael Huth," Economic Impact of IoT Cyber Risk - Analysing past and present to predict the future developments in IoT risk analysis and IoT cyber insurance", Living in the Internet of Things: Cybersecurity of the IoT, IEEE,2018.

12. Stefano Tedeschi, Christos Emmanouilidis, Jörn Mehnen ,Rajkumar Roy," A Design Approach to IoT Endpoint Security for Production Machinery Monitoring",Sensors,MDPI,2019.

13. Jiangchuan Liu,Feng Wang,2Xiaoqiang Ma, and Zhe Yang, "Recent Advances in Wireless Communication Protocols for Internet of Things" ,Hindawi Wireless Communications and Mobile Computing, WILEY,2017.

14. Rob van Kranenburg, Alex Bassi, "IoT Challenges", "IoT Challenges," Communications in Mobile Computing", A springer open,2012.

15. S. M. Riazul Islam, Daehan Kwak, MD. Humaun Kabir, Mahmud Hossain ; Kyung-Sup Kwak, "The internet of things for health care: a comprehensive survey," vol. 3, IEEE Access, 2015.

16. Hironori Washizaki, Tian Xia, Hideyuki Kanuka, Dan Yamaoto, Takao Okubo," Taxonomy and Literature Survey of Security Pattern Research", IEEE Conference on Applications, Information and Network Security (AINS),2018.

17. Roberto Ortiz,Javier Garzás,Eduardo Fernández-Medina," Analysis of Application of Security Patterns to Build Secure Systems", International Conference on Advanced Information Systems Engineering, Springer,2011 and Part of the Lecture Notes in Business Information Processing book series (LNBIP, volume 83).

18. Eduardo B. Fernandez, Hironori Washizaki, Nobukazu Yoshioka," Using Security Patterns to Develop Secure Systems-Ten Years Later", International Journal of Systems and Software Security and Protection Volume 9, Issue 4, October-December, IGI Global,2018.

19. Ishfaq Ali, Muhammad Asif,Muhammad Shabaz,Adnan Khalid, "Text Categorization Approach for Secure Design Pattern Selection Using Software Requirement Specification", Volume 6, IEEE Access, 2018.

20. Phu X. Maia, Arda Goknil, Lwin Khin Shar, Fabrizio Pastore, Lionel C. Briand," Modeling Security and Privacy Requirements: a Use Case-Driven Approach", Information and Software Technology, Elsevier, 2018.

21. Masatoshi Yoshizawa, Hironori Washizaki, Yoshiaki Fukazawa, Takao Okubo, Haruhiko Kaiya, Nobukazu Yoshioka," Implementation Support of Security Design Patterns Using Test Templates", https://www.mdpi.com/journal/information,2016.

22. M.Riaz,J.King,J.Slankas,andL.Williams, "Hidden in plain sight:Automatically identifying security requirements from natural language artifacts," 22nd International Requirements Engineering(RE) conference, IEEE,2014.

23. Alessio Ferrari, Giorgio Spagnolo, and Stefenia Gnesi, "Towards a dataset for natural language requirements processing," in Proceedings of CEUR Workshop, 2017 and also available ISTI Open portal.

24. Markus Schumacher, Eduardo Fernandez-Buglioni, Duane Hybertson, Frank Buschmann, Peter Sommerlad ,"Security Patterns: Integrating Security and Systems Engineering", John Wiley \& Sons Ltd, Chichester, UK, 2006.

25. Eduardo Fernandez-Buglioni, "Security Patterns in Practice: Designing Secure Architectures Using Software Patterns", Wiley publishing,ACM, 2013.

26. Chad Dougherty, Kirk Sayre, Robert C. Seacord, David Svoboda, Kazuya Togashi," Secure Design Patterns", CERT Program, http://www.cert.org/ , software engineering Institute,2009.

27. Exception Manager Pattern, https://www.blackhat.com/presentations/bh-federal-03/bh-fed-03peterson-up.pdf. 


\section{AUTHORS PROFILE}

Mrs. E..R.Aruna, is working as Associate Professor in Department of Information Technology, Vardhaman College of Engineering. She is pursuing her $\mathrm{PhD}$ in Computer Science and Engineering in JNTUH, Hyderabad, India. She received Master if Information Technology in 2008, Sathyabhama University, Chennai, India and received Bachelor of Computer Science and Information Technology in 2004, SITAM, Chittoor, AP, India.

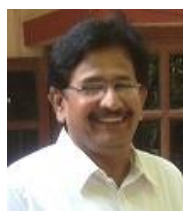

Dr. A. Rama Mohan Reddy is a Professor in the Computer Science and Engineering division at Sri Venkateswara University College of Engineering.

His research interests include Software Architecture, Software Engineering, Data Mining and optimising compilers. He received his B.Tech. from JNT University, Hyderabad in 1986, M. Tech degree in Computer Science from National Institute of Technology in 2000 Warangal and Ph. D in Computer Science and Engineering in 2007 from Sri Venkateswara University, Tirupathi, Andhra Pradesh, India.

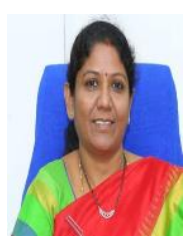

Dr. K. V. N. Sunitha, is working as Principal and Professor of CSE in BVRIT for Women, Hyderabad, India. She has done her B. Tech (ECE) from Nagarjuna University, M. Tech Computer Science from REC Warangal. She completed her Ph.D from JNTU, Hyderabad in 2006. 